\title{
Single-Crystal Nanoribbons, Nanotubes and Nanowires from Intramolecular Charge-Transfer Organic Molecules
}

\author{
Xiujuan Zhang ${ }^{l}$, Xiaohong Zhang ${ }^{1 *}$ Kai Zou $^{l}$, Chunsing Lee ${ }^{2}$, Shuittong Lee I, $^{*}$
}

${ }^{1}$ Nano-organic Photoelectronic Laboratory, Technical Institute of Physics and Chemistry, Chinese Academy of Science, Beijing 100101, China

${ }^{2}$ Center of Super-Diamond and Advanced Film (COSDAF) \& Department of Physics and Materials Science, City University of Hong Kong, Hong Kong SAR,

China

\section{Supporting Information}

\section{Part S1}

The X-ray powder diffraction pattern of the powder, nanoribbons, nanotubes and nanowires are all indexed with program Powder-X.

(1) Indexing results of the powder diffraction pattern were shown below.

The crystal structure is tetragonal.

\begin{tabular}{cccccccc} 
A & \multicolumn{1}{c}{ B } & C & ALPHA & BETA & GAMMA \\
23.74880 & 23.74880 & 6.38043 & 90.000 & 90.000 & 90.000 \\
VOLUME & $(\mathrm{A} * * 2)$ & $: 3598.59899$ & & & \\
& & & & & & \\
$\mathrm{H}$ & $\mathrm{K}$ & $\mathrm{L}$ & $\mathrm{TH}(\mathrm{OBS})$ & $\mathrm{TH}-\mathrm{ZERO}$ & $\mathrm{TH}(\mathrm{CALC})$ & DIFF. \\
1 & 1 & 0 & 2.63333 & 2.63170 & 2.62910 & 0.00260 \\
2 & 0 & 0 & 3.71593 & 3.71430 & 3.71942 & -0.00512 \\
2 & 1 & 0 & 4.15814 & 4.15651 & 4.15917 & -0.00266 \\
2 & 2 & 0 & 5.27580 & 5.27417 & 5.26376 & 0.01040 \\
3 & 2 & 0 & 6.72216 & 6.72053 & 6.71594 & 0.00459 \\
1 & 0 & 1 & 7.17531 & 7.17368 & 7.18130 & -0.00763 \\
3 & 3 & 0 & 7.90647 & 7.90484 & 7.90964 & -0.00480 \\
4 & 2 & 0 & 8.34045 & 8.33882 & 8.34047 & -0.00164
\end{tabular}




$\begin{array}{ccccccc}5 & 0 & 0 & 9.33858 & 9.33695 & 9.33325 & 0.00370 \\ 5 & 1 & 0 & 9.52728 & 9.52565 & 9.51978 & 0.00586 \\ 5 & 2 & 0 & 10.05926 & 10.05762 & 10.05942 & -0.00179 \\ 3 & 3 & 1 & 10.56682 & 10.56519 & 10.54824 & 0.01695 \\ 4 & 4 & 0 & 10.56682 & 10.56519 & 10.57262 & -0.00744 \\ 4 & 2 & 1 & 10.88414 & 10.88250 & 10.87826 & 0.00425 \\ 6 & 0 & 0 & 11.21882 & 11.21719 & 11.22204 & -0.00485 \\ 6 & 1 & 0 & 11.38120 & 11.37957 & 11.37889 & 0.00068 \\ 5 & 0 & 1 & 11.65865 & 11.65702 & 11.66443 & -0.00741 \\ 4 & 3 & 1 & 11.65865 & 11.65702 & 11.66443 & -0.00741 \\ 6 & 2 & 0 & 11.83546 & 11.83383 & 11.83762 & -0.00379 \\ 5 & 2 & 1 & 12.25743 & 12.25580 & 12.25903 & -0.00323 \\ 5 & 3 & 1 & 12.96583 & 12.96420 & 12.96623 & -0.00203 \\ 6 & 0 & 1 & 13.25011 & 13.24848 & 13.23924 & 0.00924 \\ 5 & 5 & 0 & 13.25011 & 13.24848 & 13.25893 & -0.01045 \\ 7 & 1 & 0 & 13.25011 & 13.24848 & 13.25893 & -0.01045 \\ 6 & 4 & 0 & 13.53595 & 13.53432 & 13.52645 & 0.00787 \\ 5 & 4 & 1 & 13.89349 & 13.89186 & 13.89997 & -0.00811 \\ 6 & 3 & 1 & 14.37586 & 14.37423 & 14.40842 & -0.03419 \\ 6 & 5 & 0 & 14.68092 & 14.67929 & 14.67452 & 0.00477 \\ 5 & 5 & 1 & 15.01413 & 15.01250 & 15.02181 & -0.00931 \\ 7 & 1 & 1 & 15.01413 & 15.01250 & 15.02181 & -0.00931 \\ 3 & 1 & 2 & 15.22649 & 15.22486 & 15.20890 & 0.01596 \\ 8 & 3 & 0 & 16.09665 & 16.09501 & 16.08882 & 0.00619 \\ 8 & 1 & 1 & 16.75241 & 16.75078 & 16.73976 & 0.01102 \\ 5 & 1 & 2 & 17.02431 & 17.02268 & 17.01775 & 0.00493 \\ 8 & 4 & 1 & 18.32988 & 18.32825 & 18.31421 & 0.01405 \\ 9 & 1 & 1 & 18.52652 & 18.52489 & 18.51532 & 0.00957 \\ 8 & 6 & 0 & 18.92324 & 18.92161 & 18.92637 & -0.00476 \\ & 0 & 0 & 18.92324 & 18.92161 & 18.92637 & -0.00476\end{array}$

(2) Indexing results of the diffraction pattern of nanoribbons were shown below.

The crystal structure is monoclinic.

\begin{tabular}{|c|c|c|c|c|c|}
\hline A & B & $\mathrm{C}$ & ALPHA & BETA & IMA \\
\hline 11.27923 & 16.96740 & 9.79524 & 90.000 & 102.025 & 90.000 \\
\hline VOLUME & $A * \star 2)$ & 1833.4 & & & \\
\hline
\end{tabular}

$\begin{array}{ccccccc}\mathrm{H} & \mathrm{K} & \mathrm{L} & \mathrm{TH}(\mathrm{OBS}) & \mathrm{TH}-\mathrm{ZERO} & \mathrm{TH}(\mathrm{CALC}) & \text { DIFF. } \\ 0 & 1 & 0 & 2.59993 & 2.59823 & 2.59996 & -0.00174 \\ 0 & 2 & 0 & 5.21958 & 5.21788 & 5.20530 & 0.01258 \\ 1 & 0 & 1 & 6.71844 & 6.71673 & 6.71351 & 0.00322 \\ 0 & 2 & 1 & 6.96617 & 6.96446 & 6.96002 & 0.00444 \\ 0 & 3 & 0 & 7.82784 & 7.82614 & 7.82148 & 0.00465\end{array}$




$\begin{array}{ccccccc}-2 & 0 & 1 & 8.37563 & 8.37392 & 8.39296 & -0.01903 \\ 0 & 3 & 1 & 9.07659 & 9.07488 & 9.09239 & -0.01751 \\ -1 & 0 & 2 & 9.30019 & 9.29849 & 9.28338 & 0.01511 \\ 0 & 2 & 2 & 10.61770 & 10.61599 & 10.63231 & -0.01632 \\ -2 & 0 & 2 & 10.94138 & 10.93967 & 10.93669 & 0.00298 \\ -3 & 2 & 1 & 13.13175 & 13.13004 & 13.12991 & 0.00014 \\ -3 & 1 & 2 & 13.90712 & 13.90541 & 13.90926 & -0.00384 \\ -3 & 2 & 2 & 14.65714 & 14.65543 & 14.64915 & 0.00628 \\ -3 & 3 & 2 & 15.82282 & 15.82112 & 15.81206 & 0.00906 \\ 2 & 2 & 3 & 18.42595 & 18.42424 & 18.41873 & 0.00551 \\ -1 & 2 & 4 & 19.09420 & 19.09250 & 19.09527 & -0.00277 \\ -4 & 0 & 4 & 22.29916 & 22.29745 & 22.29953 & -0.00208 \\ 4 & 5 & 2 & 24.65521 & 24.65350 & 24.65418 & -0.00067\end{array}$

(2) Indexing results of the diffraction pattern of nanotubes were shown below. The crystal structure is tetragonal.

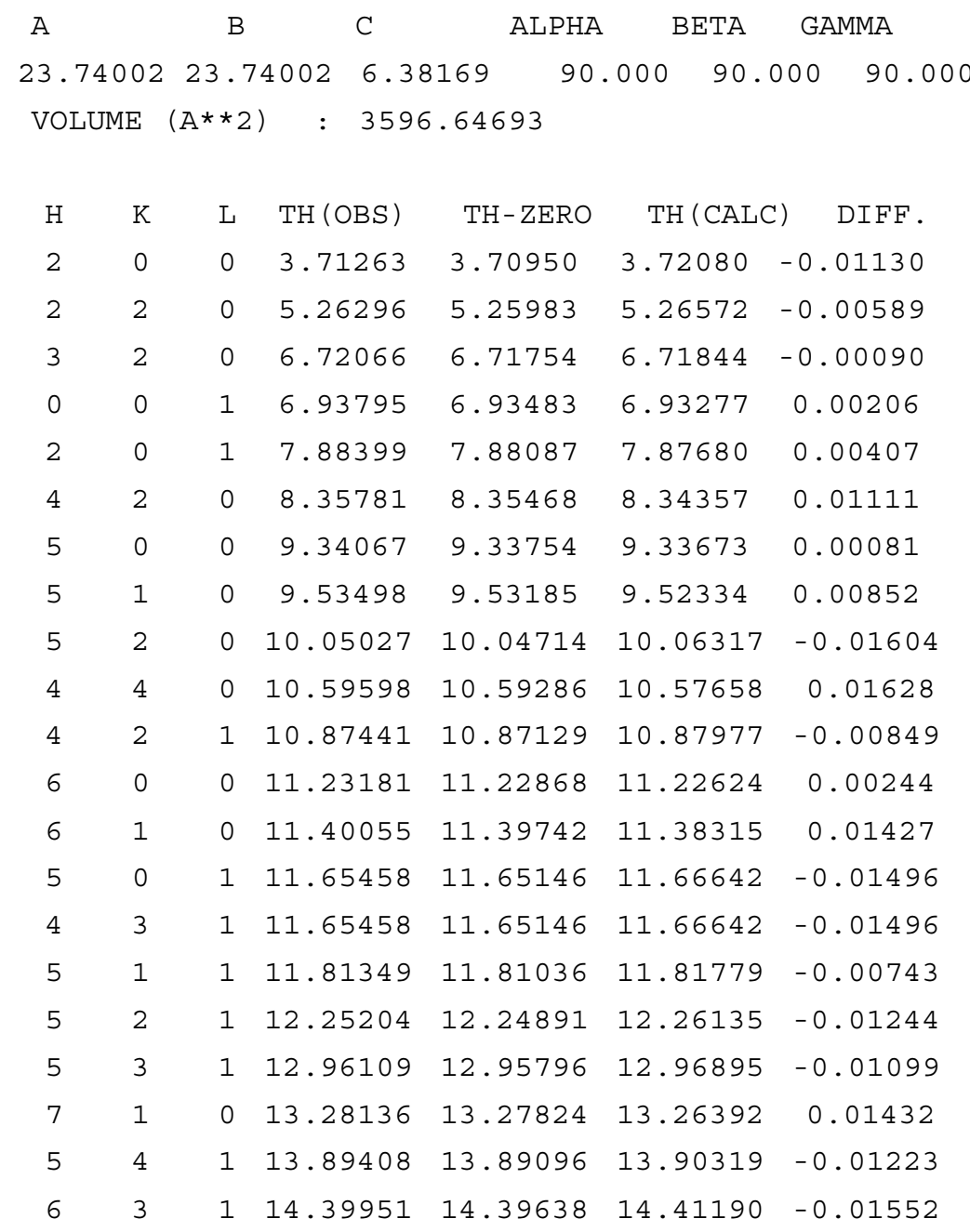




$\begin{array}{ccccccc}6 & 5 & 0 & 14.70432 & 14.70120 & 14.68006 & 0.02114 \\ 8 & 0 & 0 & 15.04458 & 15.04145 & 15.04504 & -0.00358 \\ 6 & 4 & 1 & 15.27905 & 15.27592 & 15.26479 & 0.01112 \\ 8 & 3 & 0 & 16.08697 & 16.08384 & 16.09493 & -0.01109 \\ 8 & 2 & 1 & 17.07359 & 17.07047 & 17.06960 & 0.00086 \\ 9 & 2 & 0 & 17.41559 & 17.41246 & 17.40650 & 0.00596 \\ 5 & 4 & 2 & 18.57768 & 18.57456 & 18.57242 & 0.00213 \\ 10 & 0 & 0 & 18.94336 & 18.94023 & 18.93363 & 0.00659\end{array}$

(2) Indexing results of the diffraction pattern of nanowires were shown below. The crystal structure is tetragonal.

\begin{tabular}{|c|c|c|c|c|c|}
\hline A & B & $\mathrm{C}$ & ALPHA & BETA & GAMMA \\
\hline 23.74786 & 23.74786 & 6.37239 & 90.000 & 90.000 & 90.000 \\
\hline $\mathrm{LV}$ & $* * 2)$ & 3593 & & & \\
\hline
\end{tabular}

\begin{tabular}{llllllc}
$\mathrm{H}$ & $\mathrm{K}$ & $\mathrm{L}$ & $\mathrm{TH}(\mathrm{OBS})$ & \multicolumn{4}{c}{ TH-ZERO TH(CALC) } & DIFF. \\
2 & 2 & 0 & 5.26731 & 5.25132 & 5.26397 & -0.01265 \\
3 & 2 & 0 & 6.71959 & 6.70360 & 6.71621 & -0.01261 \\
0 & 0 & 1 & 6.99138 & 6.97539 & 6.94293 & 0.03246 \\
2 & 0 & 1 & 7.90784 & 7.89185 & 7.88518 & 0.00666 \\
2 & 1 & 1 & 8.11120 & 8.09521 & 8.10403 & -0.00882 \\
4 & 2 & 0 & 8.36354 & 8.34755 & 8.34080 & 0.00676 \\
3 & 1 & 1 & 9.10202 & 9.08603 & 9.12189 & -0.03586 \\
5 & 0 & 0 & 9.36615 & 9.35016 & 9.33362 & 0.01654 \\
5 & 1 & 0 & 9.55456 & 9.53857 & 9.52016 & 0.01841 \\
5 & 2 & 0 & 10.08687 & 10.07088 & 10.05982 & 0.01106 \\
4 & 4 & 0 & 10.61271 & 10.59672 & 10.57305 & 0.02368 \\
4 & 2 & 1 & 10.90255 & 10.88656 & 10.88420 & 0.00236 \\
6 & 0 & 0 & 11.27871 & 11.26272 & 11.22249 & 0.04023 \\
5 & 0 & 1 & 11.68018 & 11.66419 & 11.67006 & -0.00587 \\
4 & 3 & 1 & 11.68018 & 11.66419 & 11.67006 & -0.00587 \\
5 & 2 & 1 & 12.26897 & 12.25298 & 12.26444 & -0.01146 \\
5 & 3 & 1 & 12.99358 & 12.97759 & 12.97143 & 0.00617 \\
7 & 1 & 0 & 13.29454 & 13.27855 & 13.25946 & 0.01908 \\
5 & 4 & 1 & 13.92475 & 13.90876 & 13.90491 & 0.00385 \\
6 & 3 & 1 & 14.43640 & 14.42041 & 14.41324 & 0.00717 \\
8 & 0 & 0 & 15.07129 & 15.05530 & 15.03995 & 0.01535
\end{tabular}




\section{Part S2}

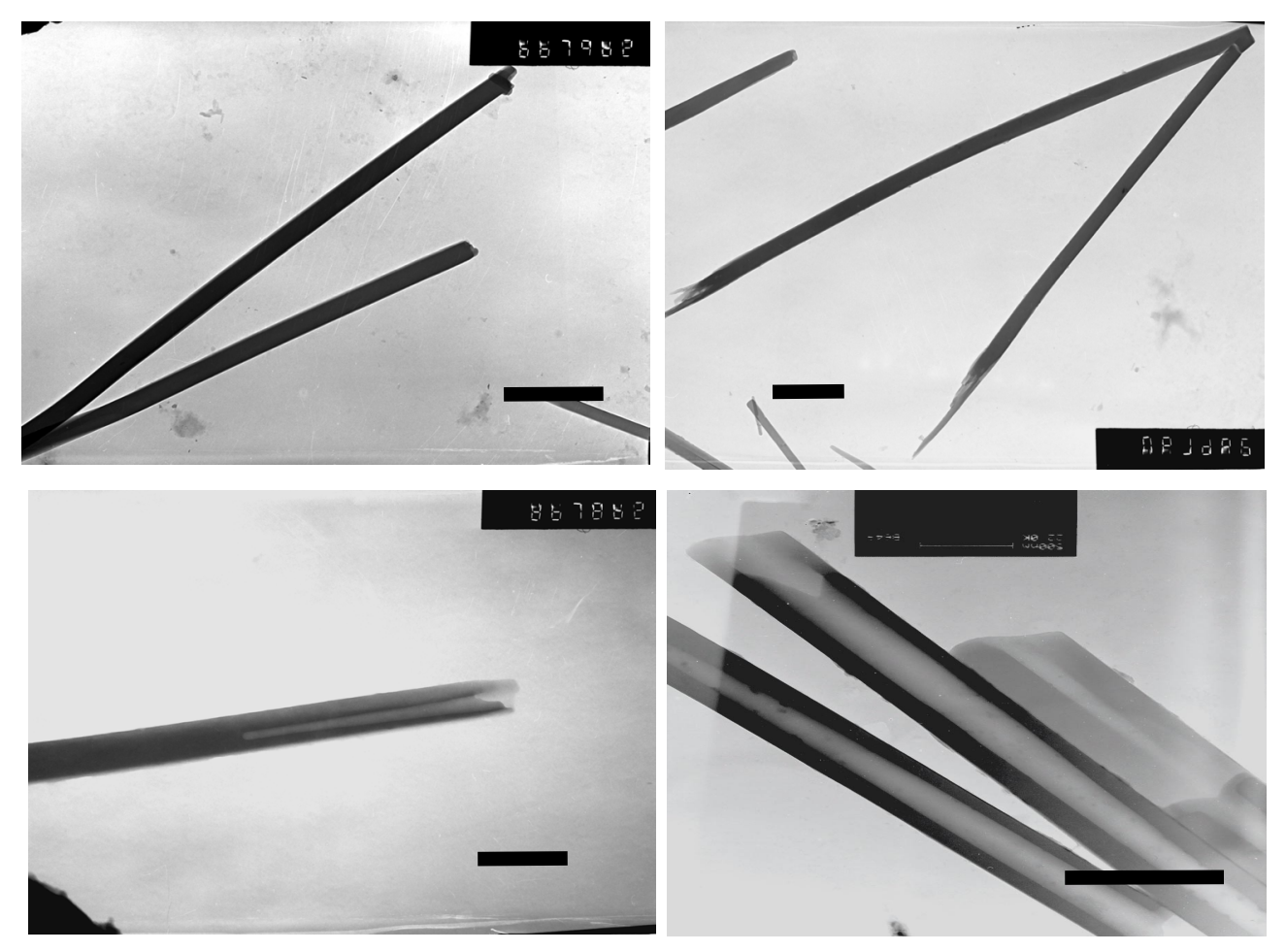

Figure S2. TEM images f DAPMP samples obtained at different time after stirring a) 1 min, b) 10 min, c) $30 \mathrm{~min}$, d) $4 \mathrm{~h}$. 


\section{Part S3}

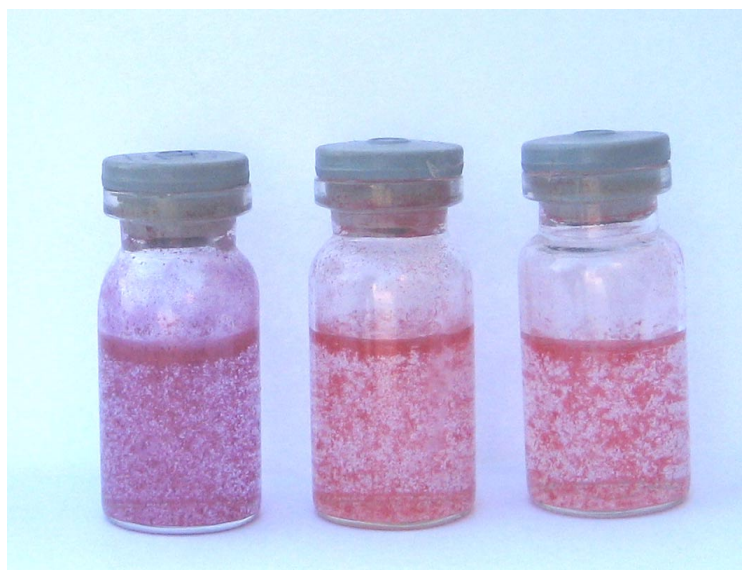

Figure S3 The image of nanoribbons (left), nanotubes (middle) and nanowires (right) respectively. 